The Astrophysical Journal, 629:979-984, 2005 August 20

(C) 2005. The American Astronomical Society. All rights reserved. Printed in U.S.A.

\title{
CONSTRAINING THE EQUATION OF STATE WITH MOMENT OF INERTIA MEASUREMENTS
}

\author{
JAMES M. LATTIMER \\ Department of Physics and Astronomy, Stony Brook University, Stony Brook, NY 11794-3800; lattimer@mail.astro.sunysb.edu \\ AND \\ Bernard F. Schutz \\ Max-Planck Institut für Gravitationsphysik, Albert-Einstein-Institut, Am Mühlenberg 1, D-14476, Potsdam, Germany; \\ bernard.schutz@aei.mpg.de \\ Received 2004 November 16; accepted 2005 April 26
}

\begin{abstract}
We estimate that the moment of inertia of star A in the recently discovered double pulsar system PSR J07373039 may be determined after a few years of observation to $\sim 10 \%$ accuracy. This would enable accurate estimates of the radius of the star and the pressure of matter in the vicinity of 1-2 times the nuclear saturation density, which would in turn provide strong constraints on the equation of state of neutron stars and the physics of their interiors.
\end{abstract}

Subject headings: binaries: close — equation of state — pulsars: general

\section{INTRODUCTION}

The discovery of the double-pulsar system PSR J0737-3039 (Burgay et al. 2003; Lyne et al. 2004) provides physicists with a remarkable laboratory for relativistic astrophysics. Besides its implications for the rate of gravitational wave bursts from neutron star coalescence (Burgay et al. 2003) and for the understanding of pulsar magnetospheres (Lyne et al. 2004), it could provide a measurement of spin-orbit coupling. Lyne et al. (2004) noted that accurate timing over a period of years could lead to a determination of the moment of inertia of star A. Spin-orbit coupling could be revealed either through an extra advancement of the periastron of the orbit above the standard post-Newtonian advance or in the precession of the orbital plane about the direction of the total angular momentum of the system (Damour \& Schaefer 1988). Given that the masses of both stars are already accurately determined by observations, a measurement of the moment of inertia of even one neutron star could have enormous importance for neutron star physics.

Despite the fact that over 1000 neutron stars have been discovered in radio and X-ray observations, and accurate masses have been determined for a dozen or so neutron stars in radio pulsar binaries (Stairs 2004), there is relatively little observational information about their radii or their interior physics. Currently, one source of candidates for revealing radii is those neutron stars for which thermal X-ray and optical radiation have been observed (see Page et al. 2004 for a review). Nevertheless, these stars have not yet provided a clear radius determination because of ambiguities due to atmospheric re-processing, interstellar absorption, and distances. Moreover, the inferred radius actually refers to the radiation radius, $R_{\infty}=R /\left(1-2 G M / R c^{2}\right)^{1 / 2}$, which is massdependent; unless independent mass measurements of the same stars become available, such as would be provided by a redshift, the radii themselves remain unknown. However, most of these sources have featureless spectra, and to date no secure redshifts have been obtained from them.

Another possibility are X-ray bursts from the surfaces of neutron stars. Recently, two lines observed in an X-ray burst spectrum have been suggested to be $\mathrm{H}$ - and He-like Fe lines, and these imply a redshift of 0.35 (Cottam et al. 2002). This inference has been given additional credibility by the detection (Villarreal \& Strohmayer 2004) of a $45 \mathrm{~Hz}$ rotational frequency for the neutron star EXO 0748-676. A low spin rate is consistent with the observed widths of these lines if their identifications with Fe are correct. Villarreal \& Strohmayer (2004) show that this consistency holds if the neutron star radius is in the range $9.5-15 \mathrm{~km}$ (corresponding to masses in the range $1.5-2.3 M_{\odot}$ ). Since this star is a member of an eclipsing binary, an independent mass measurement might be possible as well. These techniques hold promise of further constraining the radii of this star and could be extended to other X-ray bursters if redshifts from them can be observed.

At present, however, the constraints on the neutron star radius from X-ray bursters and thermal radiation from cooling neutron stars are relatively weak. We demonstrate that securing a value for the moment of inertia for a star in a radio binary pulsar system will provide important constraints on the radius of the star and the equation of state (EOS) of neutron star matter. Dimensionally, the moment of inertia is proportional to the star's mass times its radius squared, so a measurement of the moment of inertia to a given accuracy provides approximately twice that accuracy for a radius identification.

We begin in $\S 2$ by exploring the remark in Lyne et al. (2004) that the moment of inertia can be determined from measurements of spin-orbit coupling. Two observable effects of spin-orbit coupling, precession of the orbital plane and an extra contribution to the advance of the periastron, are investigated. In $\S 3$ the case of PSR J0737-3039 is investigated. We conclude that a 10\% measurement of the moment of inertia of star A in this system might be possible with observations extending over a period of a few years. In $\S 4$ we show how such a measurement leads to an estimate of the radius of the star and the density of neutron star matter in the vicinity of the nuclear saturation density. This could have crucial implications for delimiting the EOS. The conclusion, $\S 5$, compares the PSR J0737-3039 system with other relativistic binaries, and possibilities for the future of this technique are discussed.

\section{OBSERVABLE SPIN-ORBIT EFFECTS}

There are two kinds of spin-coupled precession effects in a binary system of compact stars: spin-orbit and spin-spin couplings (for a comprehensive discussion, see Barker \& O'Connell [1975] and Damour \& Schaefer [1988]). Spin-orbit coupling leads to a 
precession of the angular momentum vector $\boldsymbol{L}$ of the orbital plane around the direction of the total angular momentum $\boldsymbol{J}$ of the system. This is sometimes called geodetic precession and is related to the Thomas precession of atomic physics. Since the total angular momentum $\boldsymbol{J}=\boldsymbol{L}+\boldsymbol{S}_{\mathrm{A}}+\boldsymbol{S}_{\mathrm{B}}$ is conserved (at this order), there are compensating precessions of the spins $\boldsymbol{S}_{\mathrm{A}}$ and $\boldsymbol{S}_{\mathrm{B}}$ of the two stars. Since the orbital angular momentum dominates the spin angular momenta in binaries, the geodetic precession amplitude is very small, while the associated spin precession amplitudes are substantial. In addition to geodetic precession, spin-orbit coupling also manifests itself in apsidal motion (advance of the periastron). Spin-spin coupling is generally negligible in binary systems because $|\boldsymbol{L}| \gg\left|\boldsymbol{S}_{\mathrm{A}}\right|,\left|\boldsymbol{S}_{\mathrm{B}}\right|$.

According to Barker \& O'Connell (1975), the spin and orbital angular momenta evolve according to

$$
\begin{gathered}
\dot{\boldsymbol{S}}_{i}=\frac{G\left(4 M_{i}+3 M_{-i}\right)}{2 M_{i} a^{3} c^{2}\left(1-e^{2}\right)^{3 / 2}} \boldsymbol{L} \times \boldsymbol{S}_{i}, \\
\dot{\boldsymbol{L}}^{\mathrm{SO}}=\sum_{i} \frac{G\left(4 M_{i}+3 M_{-i}\right)}{2 M_{i} a^{3} c^{2}\left(1-e^{2}\right)^{3 / 2}}\left(\boldsymbol{S}_{i}-3 \frac{\boldsymbol{L} \cdot \boldsymbol{S}_{i}}{|\boldsymbol{L}|^{2}} \boldsymbol{L}\right),
\end{gathered}
$$

where superscript "SO" refers to the spin-coupling contribution only (there are also first- and second-order post-Newtonian terms, $1 \mathrm{PN}$ and $2 \mathrm{PN}$, respectively, unrelated to the spins, that contribute to this order). Here $a$ is the semimajor axis of the effective onebody orbital problem (sum of the semimajor axes of the two stellar orbits), $e$ is the eccentricity, and $M_{i}$ and $M_{-i}$ refer to the masses of the two binary components (we use the notation that $i=\mathrm{A}, \mathrm{B}$ and $-i=\mathrm{B}, \mathrm{A}$ ). To this order, one may employ the Newtonian relation for the orbital angular momentum:

$$
|\boldsymbol{L}|=\frac{2 \pi}{P} \frac{M_{\mathrm{A}} M_{\mathrm{B}} a^{2}\left(1-e^{2}\right)^{1 / 2}}{M}=M_{\mathrm{A}} M_{\mathrm{B}} \sqrt{\frac{G a\left(1-e^{2}\right)}{M}},
$$

where $P$ is the orbital period and $M=M_{\mathrm{A}}+M_{\mathrm{B}}$. Then, from equation (1), the spin precession periods are

$$
P_{p, i}=\frac{2 c^{2} a P M\left(1-e^{2}\right)}{G M_{-i}\left(4 M_{i}+3 M_{-i}\right)},
$$

which are not identical for the two components unless they are of equal mass. Note that the spin precession periods are independent of the spins. Also note that if the spins are parallel to $\boldsymbol{L}$, (1) there is no spin precession and (2) the spin-orbit contribution to the advance of the periastron is in a sense opposite to the direction of motion.

The spin precession leads to two observable effects. First, as the spin axes change orientation in space, the pulsar beams will sweep through changing directions in space. In many cases, this will lead to the periodic appearance and disappearance of the pulsar beams from the Earth. Second, since total angular momentum is conserved (to this order), the orbital plane will change orientation. This will be observed as a change in the inclination angle $i$.

Damour \& Schaefer (1988) have considered the question of how these effects influence the timing of binary pulsars. For the change in inclination, they find

$$
\frac{d i}{d t}=\frac{G}{a c^{2}} \frac{\pi}{\left(1-e^{2}\right)^{3 / 2}} \sum_{i} \frac{I_{i}\left(4 M_{i}+3 M_{-i}\right)}{M_{i} a^{2} P_{i}} \sin \theta_{i} \cos \phi_{i},
$$

where $\theta_{i}$ is the angle between $\boldsymbol{S}_{i}$ and $\boldsymbol{L}$ and $\phi_{i}$ is the angle between the line of sight to pulsar $i$ and the projection of $\boldsymbol{S}_{i}$ on the orbital plane. These angles follow the convention of Jenet \& Ransom (2004), but other references employ $\phi_{i}-90^{\circ}$ in place of $\phi_{i}$ (cf. Wex \& Kopeikin 1999). Also, we used $\left|\boldsymbol{S}_{i}\right|=2 \pi I_{i} / P_{i}$ where $I_{i}$ and $P_{i}$ are the moment of inertia and the spin period, respectively, of component $i$. If we concentrate on the case in which the spin of one component is much greater than that of the other, $\left|\boldsymbol{S}_{\mathrm{A}}\right| \gg\left|\boldsymbol{S}_{\mathrm{B}}\right|$, the amplitude of the precession of the inclination of the orbital plane is given by the change in $\boldsymbol{L}$ needed to compensate changes in $S_{\mathrm{A}}$. We define the angle between $\boldsymbol{L}$ and $\boldsymbol{J}$ to be $\theta-\lambda$ and the angle between $\boldsymbol{S}_{\mathrm{A}}$ and $\boldsymbol{J}$ to be $\lambda$. Since $\left|\boldsymbol{S}_{\mathrm{A}}\right| \ll|\boldsymbol{L}|$, one has $|\theta-\lambda| \ll|\lambda|$. Using the fact that $\boldsymbol{J} \simeq \boldsymbol{L}+$ $\boldsymbol{S}_{\mathrm{A}}$, one finds $|\boldsymbol{L}| \sin (\theta-\lambda) \simeq\left|\boldsymbol{S}_{\mathrm{A}}\right| \sin \lambda \simeq\left|\boldsymbol{S}_{\mathrm{A}}\right| \sin \theta$. Thus, the amplitude of the change in the orbital inclination angle $i$ due to pulsar A's precession will be

$$
\delta_{i}=\frac{\left|\boldsymbol{S}_{\mathrm{A}}\right|}{|\boldsymbol{L}|} \sin \theta_{\mathrm{A}} \simeq \frac{I_{\mathrm{A}} M}{a^{2} M_{\mathrm{A}} M_{\mathrm{B}}\left(1-e^{2}\right)^{1 / 2}} \frac{P}{P_{\mathrm{A}}} \sin \theta_{\mathrm{A}} .
$$

This will cause a periodic departure from the expected time of arrival of pulses from pulsar A of amplitude

$$
\delta t_{\mathrm{A}}=\frac{M_{\mathrm{B}}}{M} \frac{a}{c} \delta_{i} \cos i=\frac{a}{c} \frac{I_{\mathrm{A}}}{M_{\mathrm{A}} a^{2}} \frac{P}{P_{\mathrm{A}}} \sin \theta_{\mathrm{A}} \cos i,
$$

if one assumes that the orbital eccentricity is small.

For the advance of the periastron, the ratio of the spin-orbit and $1 \mathrm{PN}$ contributions is (Damour \& Schaefer 1988)

$$
\begin{aligned}
\frac{A_{p}}{A_{1 \mathrm{PN}}}= & -\frac{P}{6\left(1-e^{2}\right)^{1 / 2} M a^{2}} \sum_{i} \frac{I_{i}\left(4 M_{i}+3 M_{-i}\right)}{M_{i} P_{i}} \\
& \times\left(2 \cos \theta_{i}+\cot i \sin \theta_{i} \sin \phi_{i}\right) .
\end{aligned}
$$

In the case that $\left|S_{\mathrm{A}}\right| \gg\left|\boldsymbol{S}_{\mathrm{B}}\right|$, only the $i=A$ term contributes substantially. For comparison, the ratio of the $2 \mathrm{PN}$ to $1 \mathrm{PN}$ contributions is (Damour \& Schaefer 1988)

$$
\begin{aligned}
\frac{A_{2 \mathrm{PN}}}{A_{1 \mathrm{PN}}}= & \frac{G M}{4 a c^{2}} \sum_{i}\left\{\left[27+6 \frac{M_{i}}{M}+6\left(\frac{M_{i}}{M}\right)^{2}\right]\right. \\
& \left.\times\left(1-e^{2}\right)^{-1}-1-\frac{46 M_{i}}{3 M}+\frac{10}{3}\left(\frac{M_{i}}{M}\right)^{2}\right\} \\
\simeq & \frac{G M}{12 a c^{2}}\left(\frac{189}{1-e^{2}}-47\right)
\end{aligned}
$$

where both $i=\mathrm{A}$ and $i=\mathrm{B}$ terms contribute. The third line of equation (9) is valid in the case that $M_{\mathrm{A}}=M_{\mathrm{B}}$.

In $\S 4$ we demonstrate that a very useful constraint on the EOS can be made if the moment of inertia can be determined to about $10 \%$. In practice, it is expected that the binary components will have approximately equal masses but differing spin periods. Therefore, the spin-orbit effects will be dominated by the more rapidly rotating star, A. Besides being functions of the parameters $M_{\mathrm{A}}, M_{\mathrm{B}}, P, a, e$, and $i$, the observables $\delta t_{\mathrm{A}}$ and $A_{p}$ are also functions of $\theta_{\mathrm{A}}$ and $\phi_{\mathrm{A}}$. Therefore, extraction of $I_{\mathrm{A}}$ from these observables requires that additional information about the orientation of the spin of star A be available. Fortunately, if star $\mathrm{A}$ is observed as a pulsar, observations of the beam geometry and its precession can provide this information.

\section{APPLICATION TO PSR J0737-3039}

The observational parameters for the system PSR J07373039 are summarized in Lyne et al. (2004): $M_{\mathrm{A}} \simeq 1.34 M_{\odot}$, 
$M_{\mathrm{B}} \simeq 1.25 M_{\odot}, a / c=2.93 \mathrm{~s}, e \simeq 0.088, P_{\mathrm{A}} \simeq 22.7 \mathrm{~ms}, P_{\mathrm{B}} \simeq$ $2.77 \mathrm{~s}$, and $P \simeq 0.102$ days. We therefore observe that $P_{p \mathrm{~A}} \simeq$ $74.9 \mathrm{yr}$ and $P_{p \mathrm{~B}} \simeq 70.6 \mathrm{yr}$. With these parameters, we can form the useful combinations

$$
\begin{aligned}
\frac{G M}{a c^{2}} & =4.32 \times 10^{-6}, \\
\frac{I_{\mathrm{A}}}{M a^{2}} & =\left(4.00 \times 10^{-11}\right) I_{\mathrm{A}, 80}, \quad \frac{P}{P_{\mathrm{A}}}=3.88 \times 10^{5},
\end{aligned}
$$

where $I_{\mathrm{A}, 80}=I_{\mathrm{A}} /\left(80 M_{\odot} \mathrm{km}^{2}\right)$ scales $I_{\mathrm{A}}$ to a typical value for the moment of inertia (see $\S 4$ ).

Since $P_{\mathrm{B}} / P_{\mathrm{A}}=122$, we can ignore contributions to the precession from pulsar $\mathrm{B}$. The orientation of the spin axis of $\mathrm{A}$ relative to the orbital plane has been estimated through modeling of the intensity variations of pulsar B due to illumination by emission from A (Jenet \& Ransom 2004). There are two solutions to this model: solution 1 with $\theta_{\mathrm{A}}=13^{\circ} \pm 10^{\circ}$ (or $167^{\circ} \pm 10^{\circ}$ ) and $\phi_{\mathrm{A}}=246^{\circ} \pm 5^{\circ}$, and solution 2 with $\theta_{\mathrm{A}}=$ $\pm 90^{\circ} \pm 10^{\circ}$ and $\phi_{\mathrm{A}}=239^{\circ} \pm 2^{\circ}$. However, solution 1 is preferred on two grounds: it is improbable that a supernova kick would result in a spin axis that is so strongly misaligned with the orbital angular momentum, and solution 2 is also inconsistent with the misalignment angle between $\boldsymbol{S}_{\mathrm{A}}$ and the magnetic dipole axis, measured to be a few degrees (Demorest et al. 2004). But recent measurements of the effects of geodetic precession (Manchester et al. 2005) seem to rule out the Jenet \& Ransom solutions, as no major profile changes in the emission of star A have yet been detected. This leaves the geometry currently unconstrained, except that the large changes predicted by Jenet \& Ransom (2004) are ruled out. Similarly, the Demorest work uses polarization data of pulsar A to determine the viewing geometry, but the rotating vector model only poorly models the position angle (Ramachandran et al. 2004). However, one can still expect that the emission profiles will change eventually, leading to a determination of the orbital geometry. Also, it may be possible to get independent geometry information from the light-bending effect (Doroshenko \& Kopeikin 1995; Kramer et al. 2005b) and the interaction for the pulsar radiation between $\mathrm{A}$ and B. For the purpose of discussion, however, we assume solution 1 of Jenet \& Ransom, even though our estimates will eventually have to be modified. We expect no qualitative changes in our conclusions. The orientation of B has not been estimated so far, but this is irrelevant since the spin of pulsar B can be ignored.

Recent determinations of the inclination angle are $i=$ $88.4_{-1.4}^{+1.6} \mathrm{deg}$ (Ransom et al. 2004) and $i=90.26 \pm 0.13 \mathrm{deg}$ (Coles et al. 2005) from scintillation. However, the latter determination is not consistent with recent radio observations that yield $i=87.9_{-0.6}^{+0.7}$ deg (Kramer et al. 2005a). Since the scintillation results may be affected by unaccounted refraction effects (see discussion in Coles et al. 2005), we employ the results of Kramer et al. (2005a) here. The facts that the orbit is seen nearly edge-on and that $S_{\mathrm{A}}$ is only slightly misaligned from $\boldsymbol{L}$ makes this a special case in which the amplitude of the timing change produced by the orbital plane precession will be extremely small, $\delta t_{\mathrm{A}} \simeq(0.7 \pm 0.6) I_{\mathrm{A}, 80} \mu \mathrm{s}$. Not only is the magnitude very small, but the large relative uncertainties in both $\cos i(\cos i \simeq$ $\left.0.037_{-0.013}^{+0.010}\right)$ and in $\sin \theta_{\mathrm{A}}\left(\sin \theta_{\mathrm{A}} \simeq 0.22 \pm 0.17\right)$ combine to give a huge uncertainty in $\delta t_{\mathrm{A}}$. Current technology allows timing accuracies at the level of tenths of microseconds, limited by the quality of clocks and the characterization of radio antenna signal paths (Lorimer 2004). Given this sensitivity, and the current uncertainties in the observed angles, observation of the changing inclination due to orbital precession seems marginal. Even if timing sensitivities to the order of $0.01 \mu$ s were possible, errors in both $i$ and $\theta_{\mathrm{A}}$ would have to be substantially reduced in order to measure $I_{\mathrm{A}}$ to the order of $10 \%$.

The periastron advance due to spin-orbit coupling, being proportional to $\sin i$ and $\cos \theta_{\mathrm{A}}$, is on the other hand less sensitive to errors in these angles. In addition, since $i \simeq 90^{\circ}$, equation (8) shows that uncertainties in the angle $\phi_{\mathrm{A}}$ become largely irrelevant. The $1 \mathrm{PN}$ periastron advance is

$$
A_{1 \mathrm{PN}}=\frac{6 \pi}{1-e^{2}} \frac{G M}{a c^{2}} \operatorname{rad} \text { orbit }^{-1},
$$

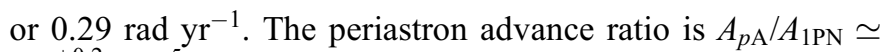
$3.4_{-0.1}^{+0.2} 10^{-5} I_{\mathrm{A}, 80}$. In practice, to measure the spin-orbit effect to $10 \%$ means subtracting out the spinless pieces of the periastron precession $\left(A_{1 \mathrm{PN}}\right.$ and $\left.A_{2 \mathrm{PN}}\right)$ to about 1 part in $10^{5}$. Fortunately, both $a \sin i$ and $P$ are well known and will be even better known in the future, and the spinless periastron advance depends on only the total mass, not the individual masses. The total mass $M=a^{3}(2 \pi / P)^{2}$ is known to the accuracy of $\sin i$. But $\sin i$ is currently known to about 4 parts in $10^{4}$, using the determination of Kramer et al. (2005a), and its accuracy will also improve with time. (Note that the estimate of Coles et al. [2005] would have resulted in an error in $\sin i$ of 2 parts in $10^{5}$.) But the spin-orbit contribution depends on the individual masses, requiring for our purposes the determination of essentially three post-Newtonian parameters, as Damour \& Schaefer (1988) have emphasized, to the accuracy of 1 part in $10^{5}$.

We do not as yet have a third observable parameter with anything approaching the required accuracy. The accuracy of $a_{\mathrm{B}} \sin i$ at present is a few parts in $10^{3}$. If the refinement of this parameter should only increase linearly inversely with the duration of observations, the necessary accuracy could not be achieved within a few years. However, the fact that one will be observing the orbits of the pulsars from essentially all sides due to the large precession might allow a large enough precision in the measurement of the size of B's orbit. Another possibility would be a precision measurement of the parameter $\gamma$, but this will be more difficult in terms of how its measurement precision scales with time. Even better would be a precise VLBI measurement for the parallax and proper motion (in progress), which could yield an accurate value of $\dot{P}_{b}$ (due to gravitational radiation reaction), because its precision should scale with time to the -2.5 power. A further reason to be optimistic is that current measurements rely mostly on a mixture of Parkes and some Green Bank Telescope (GBT) data. With additional, much more precise GBT data coming in, one will be in a position to obtain a big jump in precision. Moreover, even if $\gamma$, the mass ratio, and $\dot{P}_{b}$ measurements might not be so precise on their own, they will trace out some common contour limits that may yield the precision to within an adequate confidence limit.

The epoch of periastron changes due to spin-orbit coupling by about $0.014 \mathrm{~s} \mathrm{yr}^{-1}$. For comparison, the second postNewtonian correction contributes a periastron advance time of about $0.02 \mathrm{~s} \mathrm{yr}^{-1}$ and must therefore be included in the pulsar timing model, but this is straightforward (Lyne et al. 2004; Kramer et al. 2005b).

The determination of $I_{\mathrm{A}}$ from periastron advance measurements will only be as accurate as our understanding of $\theta_{\mathrm{A}}$. As discussed above, this is not well known at present. Nevertheless, the large magnitude of the expected precession should lead to substantial improvements in our knowledge of the pulse geometry. Fortunately, we do not need to know this angle to the 


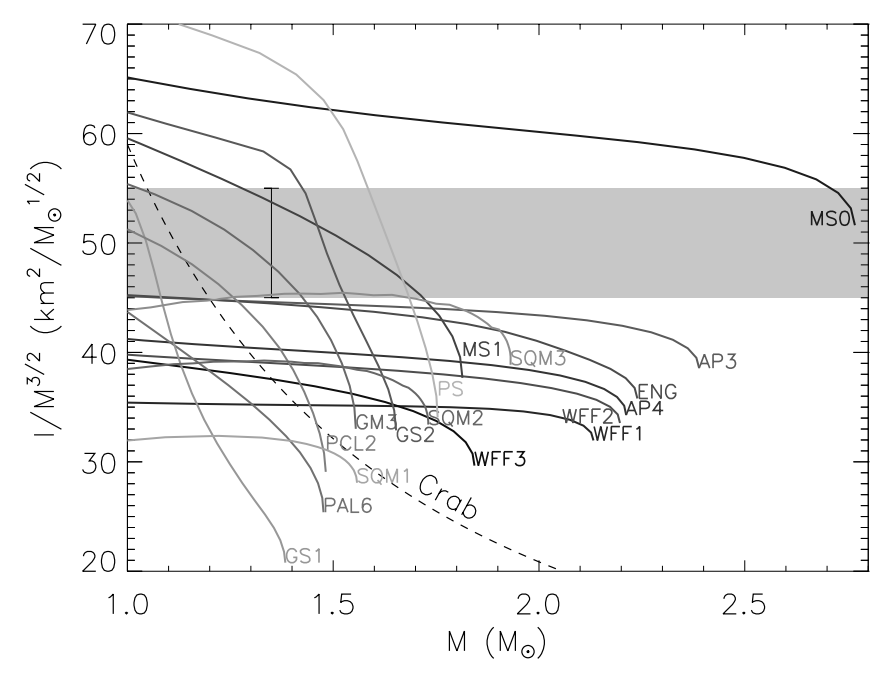

FIG. 1.- Moment of inertia scaled by $M^{3 / 2}$ as a function of stellar mass $M$. Equation-of-state labels are described in Lattimer \& Prakash (2001). The shaded band illustrates a $10 \%$ error on a hypothetical $I / M^{3 / 2}$ measurement of $50 \mathrm{~km}^{2} M_{\odot}^{-1 / 2}$; the error bar shows the specific case in which the mass is $1.34 M_{\odot}$. The dashed curve labeled "Crab" represents the lower limit derived by Bejger \& Haensel (2003) for the Crab pulsar.

tremendous precision needed for the orbital parameters. We therefore conclude that measurement of the spin-orbit periastron advance, resulting in a moment of inertia measurement to the order of $10 \%$ accuracy within several years, seems possible.

\section{CONSTRAINING THE EOS}

Estimating a neutron star's moment of inertia from timing observations of a radio binary pulsar has significant implications for constraining the EOS. In some respects, a moment of inertia measurement could be more useful than a radius measurement of the same accuracy. First, the neutron star mass in a radio binary will obviously already be known to high precision, while a radius measurement from observations of thermal radiation actually refers to the radiation radius $R_{\infty}=R /(1-$ $\left.2 G M / R c^{2}\right)^{1 / 2}$. To obtain the radius and mass separately requires an estimate of the star's redshift $\left(1-2 G M / R c^{2}\right)^{-1 / 2}-1$, which so far is not yet available for any thermal source. Moreover, the accuracy of radiation radius estimates from thermal sources is limited by uncertainties due to source atmospheric reprocessing, interstellar absorption, and distances. In the case of X-ray bursters, for the one case in which a redshift measurement may be secure, EXO 0748-676, no independent mass measurement is yet available, and radius information obtained from fitting the line profile with the observed rotation rate is relatively weak. Mass and moment of inertia measurements from radio binary pulsars are not sensitive to distance uncertainties, and a redshift is not required. Second, the range of moments of inertia for various neutron star models (including strange quark matter stars) is, in relative terms, larger than the predicted range of radii (see Fig. 1). This follows from the dimensional relation $I \propto M R^{2}$. For example, it is $30-240 M_{\odot} \mathrm{km}^{2}$ for masses $>1 M_{\odot}$ and is 53$109 M_{\odot} \mathrm{km}^{2}$ for a $1.4 M_{\odot}$ star. For comparison, the range of radii for a $1.4 M_{\odot}$ model is $9-16 \mathrm{~km}$.

Spanning the same set of equations of state as in the compendium of Lattimer \& Prakash (2001), moments of inertia for normal neutron and strange quark matter stars are displayed in Figure 1. The moments of inertia have been scaled by a factor $M^{3 / 2}$ to reduce the range of the ordinate. For most masses, the range in $I$ is approximately a factor of $2-3$. The significance of

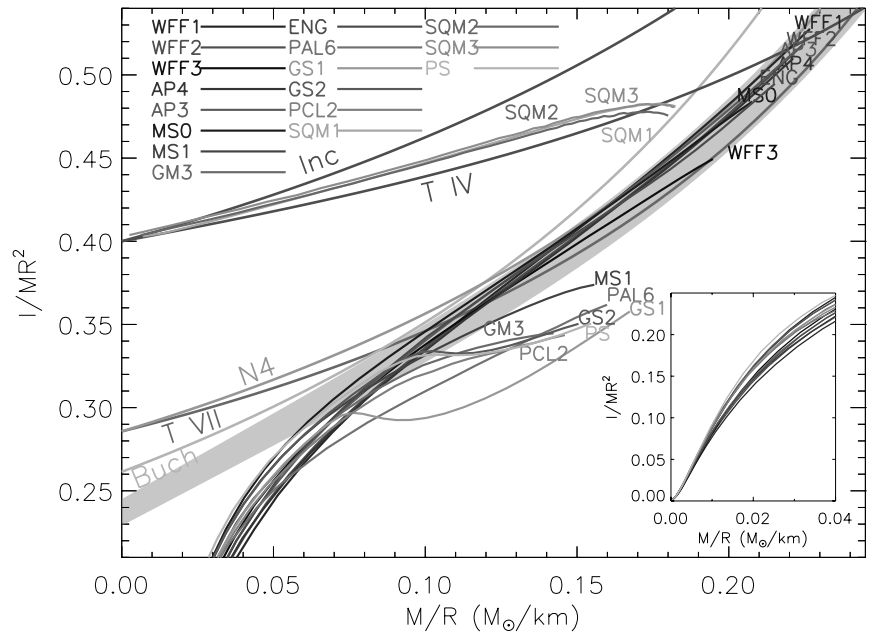

FIG. 2.-Moment of inertia as a function of the relativity parameter $M / R$. The curves labeled TIV, TVII, NIV, Inc, and Buch refer to analytic solutions of Einstein's equations, while other curves refer to a variety of equations of state (see Lattimer \& Prakash 2001 for details). The shaded band illustrates the relation eq. (12).

a measurement of $I$ with $\pm 10 \%$ accuracy is illustrated by the shaded band centered on the hypothetical measurement, here taken to be $I / M^{3 / 2}=(50 \pm 5) \mathrm{km}^{2} M_{\odot}^{-1 / 2}$, together with an error bar located at a precisely measured mass, taken to be $1.34 M_{\odot}$. It is clear that relatively few equations of state would survive these constraints. Those families of models lying close to the measured values would have their parameters limited correspondingly.

If the EOS does not have a large degree of softening at supernuclear densities, possibly introduced by hyperons, Bose condensates or self-bound strange quark matter, a moment of inertia determination furthermore permits one to estimate the neutron star radius to a relative uncertainty smaller than the relative uncertainty in the moment of inertia measurement. Figure 2 shows the moment of inertia as a function of the relativity parameter $M / R$ for the same equations of state displayed in Figure 1 . Several analytic solutions of Einstein's equations that are applicable to either normal or self-bound stars are displayed as well. These solutions are all scale-free and are functions of $M / R$ alone; hence, they cannot be displayed in Figure 1. Unless the EOS has an appreciable degree of softening, usually indicated by a maximum mass of order 1.6 $M_{\odot}$ or less, or unless it is self-bound, there appears to be a relatively unique relation between $I / M R^{2}$ and $M / R$. For $M / R$ values greater than approximately $0.07 M_{\odot} \mathrm{km}^{-1}$, i.e., for $M \geq 1.0 M_{\odot}$, this relation can be approximated by

$$
I \simeq(0.237 \pm 0.008) M R^{2}\left[1+4.2 \frac{M \mathrm{~km}}{M_{\odot} R}+90\left(\frac{M \mathrm{~km}}{M_{\odot} R}\right)^{4}\right]
$$

An analogous fit has also been suggested by Bejger \& Haensel (2002):

$$
I \simeq \frac{2}{9}\left(1+5 \frac{M \mathrm{~km}}{R M_{\odot}}\right)
$$

for $M / R>0.1 M_{\odot} \mathrm{km}^{-1}$; however, this fit underestimates $I$ for the largest neutron star masses.

Simultaneous mass and moment of inertia measurements could therefore usefully constrain the radius. The relevant radius 


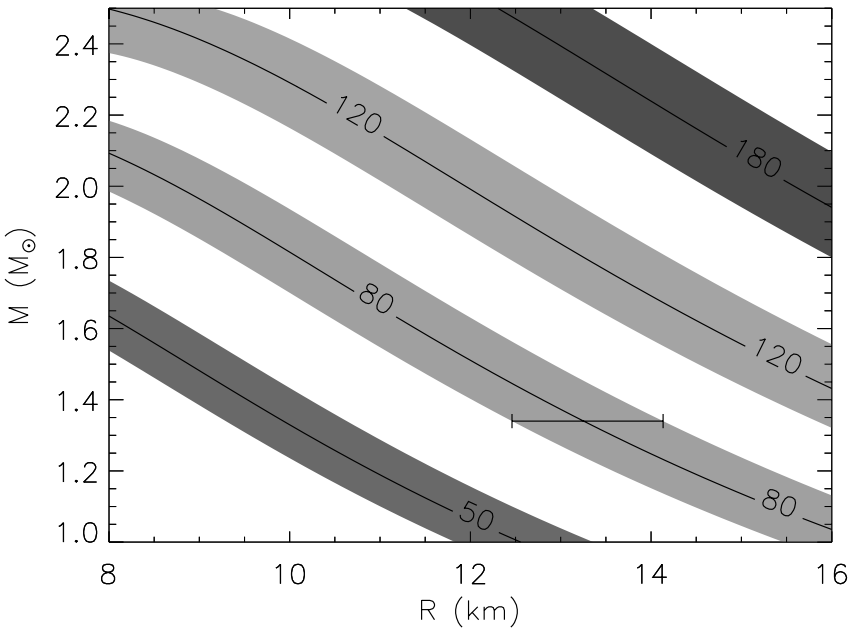

FIG. 3.- Radius limits imposed by simultaneous moment of inertia and mass measurements, established from eq. (12). Moment of inertia error bands include measurement uncertainties of $10 \%$ and systematic uncertainties from eq. (12) and are labeled in units of $M_{\odot} \mathrm{km}^{2}$. The horizontal error bar illustrates the hypothetical case in which $M$ and $I$ are measured to be $1.34 M_{\odot}$ and $80 \pm 8 M_{\odot} \mathrm{km}^{2}$, respectively.

relation can be determined by inversion of equation (12). Figure 3 shows how the radius could be constrained for selected moment of inertia measurements having $10 \%$ uncertainty. For a $1.4 M_{\odot}$ star, this typically results in a radius estimate with about $6 \%-7 \%$ uncertainty. Even in the event of significant softening of the EOS, the uncertainty of the estimated radius would be degraded by no more than a factor of 2 . Of course, the accumulation of more than a single $I-M$ pair would significantly enhance the constraints.

The importance of a radius determination is that it immediately translates into a measure of the neutron star matter pressure near the nuclear saturation density (Lattimer \& Prakash 2001). In particular, the relation between these quantities is of the form of a power law

$$
R_{M} P(n)^{-1 / 4}=C(n, M)
$$

Here $R_{M}$ is the neutron star radius in km for the mass $M$ and $P(n)$ is the matter pressure in $\mathrm{MeV} \mathrm{fm}{ }^{-3}$ evaluated at the density $n$. The constant $C$ is parameterized by both $n$ and $M$, but its dependence on $M$ is weak (approximately scaling as $M^{-1 / 8}$ ). In the case of $M=1.4 M_{\odot}$, a least-squares fit to approximately 30 equations of state yielded $C(n, 1.4)=9.30 \pm 0.60,7.00 \pm$ 0.31 , and $5.72 \pm 0.25$ for the cases $n / n_{s}=1,1.5$, and 2 , respectively. This relation could be made more precise by adjusting the exponent of $P$ (Steiner et al. 2005), but we choose not to do so here. The pressure of neutron star matter at these densities is primarily a function of the density dependence of the nuclear symmetry energy. In general, therefore, we expect that measurement of the moment of inertia would provide estimates of pressures. Figure 4 illustrates the situation for some representative equations of state for the case of $M=1.34 M_{\odot}$. It is observed that the phenomenological fits equations (12) and (14) adequately describe all but the softest equations of state (e.g., PS and PAL6) for each density. The uncertainties in the estimated pressure obtained from a measured value of the moment of inertia are moderate, amounting to a factor of about 2 when a $10 \%$ uncertainty in the moment of inertia measurement is included. Nevertheless, given the fact that present estimates of

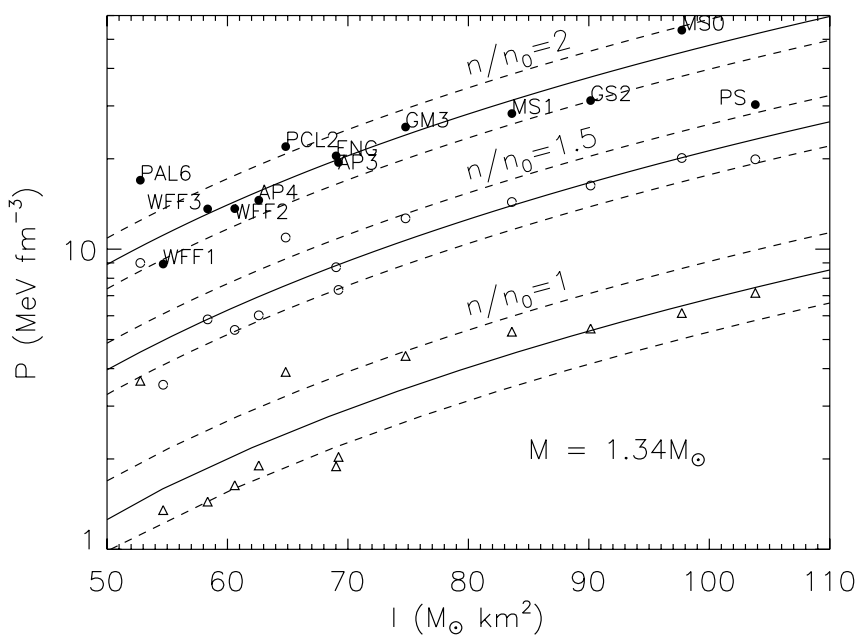

FIG. 4.-Pressures at the densities $n_{s}$ (open triangles), $1.5 n_{s}$ (open circles), and $2 n_{s}$ (filled circles) as a function of the moment of inertia for $1.34 M_{\odot}$ stars. Solid curves show the relations between $P$ and $I$ derived by combining eqs. (12) and (14). Dashed curves include the errors in these fits. Equations of states and their labels are described in Lattimer \& Prakash (2001).

the pressure of matter at the nuclear saturation density span a range of a factor of 6 , this information will be valuable.

\section{DISCUSSION AND CONCLUSIONS}

The precession of the spins and orbital plane of the pulsar binary PSR J0737-3039 occurs with a period of about $75 \mathrm{yr}$. Both the inclination of the orbit along the line of sight and the position of the periastron change due to spin-orbit coupling. The edge-on nature of this binary, coupled with the slight misalignment of $\boldsymbol{S}_{\mathrm{A}}$ and $\boldsymbol{L}$ probably precludes observation of the changing inclination angle, but the spin-orbit contribution to the advance of the periastron, which amounts to a timing residual of about $0.02 \mathrm{~s} \mathrm{yr}^{-1}$, should be measurable within a few years. In this case, the edge-on orbital plane and near alignment of $\boldsymbol{S}_{\mathrm{A}}$ and $\boldsymbol{L}$ work in favor of a precision measurement of $I_{\mathrm{A}}$. A measure of $I_{\mathrm{A}}$ to about $5 \%-10 \%$ accuracy seems possible, and significant constraints on the EOS would be forthcoming. It would also lead to

TABLE 1

Comparison of Binary Pulsars

\begin{tabular}{|c|c|c|c|}
\hline Parameter & PSR J0737-3039 & PSR B1913+16 & PSR B1534+12 \\
\hline References. & $1,2,3$ & 4,5 & $6,7,8$ \\
\hline$a / c(\mathrm{~s}) .$. & 2.93 & 6.38 & 7.62 \\
\hline$P(\mathrm{~h}) \ldots \ldots \ldots \ldots \ldots \ldots \ldots$ & 2.45 & 7.75 & 10.1 \\
\hline e & 0.088 & 0.617 & 0.274 \\
\hline$M_{\mathrm{A}}\left(M_{\odot}\right) \ldots \ldots \ldots \ldots \ldots$ & 1.34 & 1.441 & 1.333 \\
\hline$M_{\mathrm{B}}\left(M_{\odot}\right) \ldots \ldots \ldots \ldots \ldots$ & 1.25 & 1.387 & 1.345 \\
\hline$T_{\mathrm{GW}}(\mathrm{Myr}) \ldots \ldots \ldots \ldots$ & 85 & 245 & 2250 \\
\hline$i(\mathrm{deg}) \ldots \ldots \ldots \ldots \ldots \ldots$ & $87.9 \pm 0.6$ & 47.2 & 77.2 \\
\hline$P_{\mathrm{A}}(\mathrm{ms}) \ldots \ldots \ldots \ldots$ & 22.7 & 59 & 37.9 \\
\hline$\theta_{\mathrm{A}}(\mathrm{deg}) \ldots \ldots \ldots \ldots \ldots \ldots$ & $13 \pm 10$ & 21.1 & $25.0 \pm 3.8$ \\
\hline$\phi_{\mathrm{A}}(\mathrm{deg}) \ldots \ldots \ldots \ldots \ldots$ & $246 \pm 5$ & 9.7 & $290 \pm 20$ \\
\hline$P_{p \mathrm{~A}}(\mathrm{yr}) \ldots \ldots \ldots \ldots \ldots$ & 74.9 & 297.2 & 700 \\
\hline$\delta t_{a} / I_{\mathrm{A}, 80}(\mu \mathrm{s}) \ldots \ldots \ldots \ldots$ & $0.7 \pm 0.6$ & 11.2 & $7.9 \pm 1.1$ \\
\hline$A_{p \mathrm{~A}} /\left(A_{1 \mathrm{PN}} I_{\mathrm{A}, 80}\right) \ldots \ldots .$. & $3.4_{-0.1}^{+0.2} \times 10^{-5}$ & $1.0 \times 10^{-5}$ & $1.15_{-0.03}^{+0.04} \times 10^{-5}$ \\
\hline$A_{2 \mathrm{PN}} / A_{1 \mathrm{PN}} \ldots \ldots \ldots \ldots$ & $5.2 \times 10^{-5}$ & $4.7 \times 10^{-5}$ & $2.3 \times 10^{-5}$ \\
\hline
\end{tabular}

REFERENCES.-(1) Lyne et al. 2004; (2) solution 1, Jenet \& Ransom 2004; (3) Kramer et al. 2005a; (4) Weisberg \& Taylor 2002; (5) Weisberg \& Taylor 2005; (6) Stairs et al. 2002; (7) Bogdanov et al. 2002; (8) Stairs et al. 2004. 
more reliable estimates of neutron star radii and matter pressures near the nuclear saturation density than are currently available.

Previously, the moment of inertia of the Crab pulsar was estimated by Bejger \& Haensel (2003). They used an estimate (Fesen et al. 1997) for the mass of the ionized portion of the Crab's remnant, $4.6 \pm 1.8 M_{\odot}$, to infer a lower limit to the Crab pulsar's moment of inertia of $97 \pm 38 \mathrm{M}_{\odot} \mathrm{km}^{2}$. Within errors, the lower limit rules out only the softest equations of state, as is shown in Figure 1.

It should be noted that the binary PSR J0737-3039, although highly relativistic, is not favorably inclined for observation of the precession of the inclination, which is proportional to $\cos i$. But this particular inclination does allow a simplification of the interpretation of the periastron advance. Not only is the spinorbit contribution nearly independent of the pulsar orientation angle $\phi_{\mathrm{A}}$, but errors in $i$ are also not going to be a significant restriction to a measurement of $I_{\mathrm{A}}$.

It is interesting to compare PSR J0737-3039 with other known relativistic binaries. Table 1 compares properties of the relativistic binaries for which spin orientation of pulsar A can be estimated. It is noteworthy that the net timing delays caused by precessioninduced inclination shifts are more than an order of magnitude larger for PSR B1913+16 and PSR B1534+12 than for PSR J0737-3039, due to their smaller inclinations. However, the precessional periods of these two systems are 4 times larger, and their periastron advances are about 6 times smaller than for PSR J0737-3039. This factor of 24 in observability is significant and explains why measurements of this effect in these systems have not been made.

The nearness and faintness of this binary gives hope that other highly relativistic systems might eventually be observed. If further highly relativistic systems are discovered, it seems unlikely that any of them will have an inclination angle so unfortunate for detecting the precession of the orbital plane. With two spin-orbit effects to be observed, the moment of inertia might be measured to even greater accuracy than contemplated for PSR J0737-3039.

This work was supported in part by the US Department of Energy under grant DE-FG02-87ER-40317. J. M. L. appreciates the hospitality of the Albert-Einstein-Institut, where this work was initiated. Discussions with C. Cutler, T. Damour, M. Kramer, and G. Esposito-Farese were essential and are greatly appreciated. In particular, we thank M. Kramer for assistance with the interpretation of the observations of PSR J0737-3039 and the prospects for improvements.
Barker, B. M., \& O’Connell, R. F. 1975, Phys. Rev. D, 12, 329

Bejger, M., \& Haensel, P. 2002, A\&A, 396, 917 2003, A\&A, 405, 747

Bogdanov, S., Pruszunska, M., Lewandowski, W., \& Wolszczan, A. 2002, ApJ, 581,495

Burgay, M., et al. 2003, Nature, 426, 531

Coles, W. A., McLaughlin, M. A., Rickett, B. J., Lyne, A. G., \& Bhat, N. D. R. 2005, ApJ, 623, 392

Cottam, J., Paerels, F., \& Mendez, M. 2002, Nature, 420, 51

Damour, T., \& Schaefer, G. 1988, Nuovo Cimento B, 101, 127

Demorest, P., Ramachandran, R., Backer, D. C., Ransom, S. M., Kaspi, V., Arons, J., \& Spitkovsky, A. 2004, ApJ, 615, L137

Doroshenko, O. V., \& Kopeikin, S. M. 1995, MNRAS, 274, 1029

Fesen, R. A., Shull, J. M., \& Hurford, A. P. 1997, AJ, 113, 354

Jenet, F. A., \& Ransom, S. M. 2004, Nature, 428, 919

Kramer, M. et al. 2005a, in Proc. 22nd Texas Symp. on Relativistic Astrophysics, ed. P. Chen \& G. Madejski (Stanford: Stanford Univ.), in press (astro-ph/0503386) 2005b, in ASP Conf. Ser. 328, Binary Radio Pulsars, ed. F. A. Rasio \& I. H. Stairs (San Francisco: ASP), 59

Lattimer, J. M., \& Prakash, M. 2001, ApJ, 550, 426

\section{REFERENCES}

Lorimer, D. R. 2004, Living Rev. Relativ., 4, 5, http:/www.livingreviews.org/ lrr-2001-5

Lyne, A. G., et al. 2004, Science, 303, 1153

Manchester, R. N., et al. 2005, ApJ, 621, L49

Page, D., Lattimer, J. M., Prakash, M., \& Steiner, A. W. 2004, ApJS, 155, 623

Ramachandran, R., Backer, D. C., Demorest, P., Ransom, S. M., \& Kaspi, V. M. 2004, ApJ, submitted (astro-ph/0404392)

Ransom, S. M., Kaspi, V. M., Ramachandran, R., Demorest, P., Backer, D. C., Pearl, E. D., Ghigo, F. D., \& Kaplan, D. L. 2004, ApJ, 609, L71

Stairs, I. H. 2004, Science, 304, 547

Stairs, I. H., Thorsett, S. E., \& Arzoumanian, Z. 2004, Phys. Rev. Lett, 93, 141101

Stairs, I. H., Thorsett, S. E., Taylor, J. H., \& Wolszczan, A. 2002, ApJ, 581, 501

Steiner, A. W., Prakash, M., Lattimer, J. M., \& Ellis, P. J. 2005, Phys. Rep., 411,325

Villarreal, A. R., \& Strohmayer, T. E. 2004, ApJ, 614, L121

Weisberg, J. M., \& Taylor, J. H. 2002, ApJ, 576, 942

2005, in ASP Conf. Ser. 328, Binary Radio Pulsars, ed. F. A. Rasio \&

I. H. Stairs (San Francisco: ASP), 25

Wex, N., \& Kopeikin, S. M. 1999, ApJ, 514, 388 